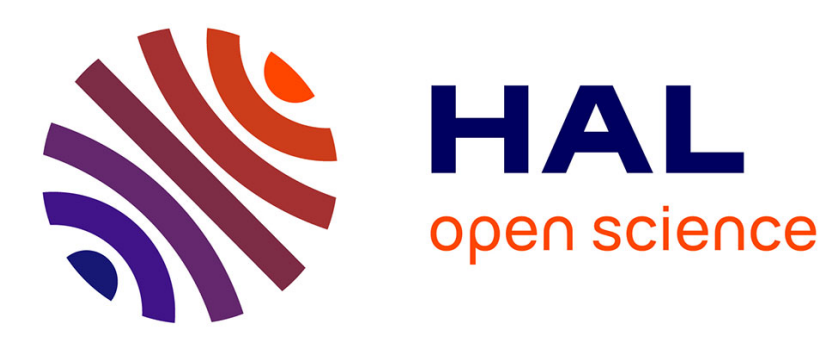

\title{
Shaping convex edges in borosilicate glass by single pass perforation with an Airy beam
}

David Sohr, Jens Ulrich Thomas, Stefan Skupin

\section{To cite this version:}

David Sohr, Jens Ulrich Thomas, Stefan Skupin. Shaping convex edges in borosilicate glass by single pass perforation with an Airy beam. Optics Letters, 2021, 46, pp.2529-2532. 10.1364/OL.423788 . hal-03155712v2

\author{
HAL Id: hal-03155712 \\ https://hal.science/hal-03155712v2
}

Submitted on 30 Jul 2021

HAL is a multi-disciplinary open access archive for the deposit and dissemination of scientific research documents, whether they are published or not. The documents may come from teaching and research institutions in France or abroad, or from public or private research centers.
L'archive ouverte pluridisciplinaire HAL, est destinée au dépôt et à la diffusion de documents scientifiques de niveau recherche, publiés ou non, émanant des établissements d'enseignement et de recherche français ou étrangers, des laboratoires publics ou privés. 


\title{
Shaping convex edges in borosilicate glass by single pass perforation with an Airy beam
}

\author{
David Sohr, ${ }^{1,2, *}$ Jens Ulrich Thomas, ${ }^{2}$ and Stefan SkUPIn ${ }^{1}$ (i) \\ ${ }^{1}$ Institut Lumière Matière, UMR 5306 Université Lyon 1-CNRS, Université de Lyon, Villeurbanne 69622, France \\ ${ }^{2}$ SCHOTT AG, Hattenbergstrasse 10, Mainz 55122, Germany \\ ${ }^{*}$ Corresponding author: david.sohr@univ-lyon1.fr
}

Received 5 March 2021; revised 23 April 2021; accepted 27 April 2021; posted 27 April 2021 (Doc. ID 423788 ); published 14 May 2021

\begin{abstract}
We demonstrate curved modifications with lengths of up to $2 \mathrm{~mm}$ within borosilicate glass produced by single $1030 \mathrm{~nm}$ picosecond laser shots with an Airy beam profile. Plasma ignition in the sidelobes of the beam as well as surface damage prove to be the crucial limitations for confined bulk energy deposition on a curved trajectory. A combined experimental and numerical analysis reveals optimum laser parameters for confined bulk energy deposition. This way, we achieved single pass perforation of a $525 \mu \mathrm{m}$ thick glass sheet and separation by a subsequent etching step, resulting in a well-defined convex edge down to a radius of curvature of $774 \mu \mathrm{m}$. (O) 2021 Optical Society of America under the terms of the OSA Open Access Publishing Agreement
\end{abstract}

https://doi.org/10.1364/OL.423788

For reasons reaching from practical purposes, such as improved handling and durability, to decorative considerations, processed glass is often required to have a seamed or round edge. Currently this requires an extra grinding step to obtain, for example, the most common curved shape, the so called "c-cut." For the preceding processing step of cutting the glass to shape, ultrashort pulse lasers used with beam profiles with an elongated focal volume (line focus) are increasingly replacing conventional cutting tools [1-3]. These straight-line foci can be used to modify the workpiece throughout its entire depth with one single laser shot, effectively perforating it instantaneously, instead of having to dig into the material, as, for example, by ablation [4]. Avoiding the need to remove material makes this perforation a very clean process. The most prominent beam shape in this context has been the Bessel-Gauss beam [5,6]. With its nondiffracting, self-healing character, it provides a highly uniform and robust line focus within the material. Deformation of the laser beam shape through nonlinear propagation effects can be minimized by using high NA focusing optics, feeding energy to the line focus increasingly from the side, and by employing bursts of laser pulses [1]. The laser energy deposited in a narrow channel acts to create permanent modifications reaching from structural changes to voids and cracks, in any case, weakened zones compared to the pristine material. By laterally joining several of these weak zones, e.g., by mechanical cleaving or etching, the glass can then be separated. This way single pass laser glass cutting up to a thickness of $12 \mathrm{~mm}$ has been demonstrated [3], and this thickness was fundamentally limited only by the laser pulse energy and the size of the optics. The results of such a cutting process are straight, generally vertical edges. Even though the cleaved edges with a root-mean-square roughness of $1 \mu \mathrm{m}$ resemble a fine polish, the sharp $90^{\circ}$ edge has usually to be removed in a subsequent edge shaping step.

In this Letter, we report on combining cutting and edge shaping of glass in one process. To this end, we replace the Bessel-Gauss beam in the aforementioned micromachining setup with an Airy-Gauss beam to achieve a curved edge as the result of the cutting process. The Airy beam is the most prominent nondiffracting beam following a curved trajectory, that is to say, its mainlobe appears to be accelerated in the transverse plane during propagation. After its theoretical description in 1979 [7], the optical, finite-energy Airy-Gauss beam featuring a curved line focus was first demonstrated experimentally in 2007 [8]. While the applications that followed reach from 1D light sheets [9] to light bullets formed by a ring-Airy [10], the most common case is still the $2 \mathrm{D}$ case given by the linear combination of cubic phases along two perpendicular axes in the far field $[4,11,12]$. As the first of a whole group of accelerating beams [13], the 2D Airy beam has been used before to create long, curved plasma channels (ca. $1 \mathrm{~m}$ length) in air [12].

To our knowledge there has not yet been a case of the Airy beam being used for creating extended mechanical damage zones in dense media, analogous to the Bessel beam perforation in dielectrics. Shaping convex edges with the Airy beam has been limited to ablation of diamond and silicon for a thickness of up to ca. $100 \mu \mathrm{m}$ [4], requiring many passes for a single convex profile and producing significant pollution due to the material removed during the laser process. The hard focusing (NA 0.8) in this previous study provided a strong curvature with radius $r=120 \mu \mathrm{m}$, but also limited the length of the line focus. Curved refractive index modifications produced by single shot Airy beams in glass were demonstrated up to a length of only ca. $20 \mu \mathrm{m}$ [11]. Here, we first demonstrate curved bulk modifications in borosilicate glass up to lengths in the millimeter range. Second, by means of experiments and numerical simulations, we investigate the influence of the laser parameters, in particular, pulse duration and number of pulses in a burst, to optimize the bulk energy deposition. Finally, we confirm that the optimized laser process causes sufficient mechanical damage throughout 
the glass sheet to allow subsequent separation, and report the creation of a well-defined convex edge.

We begin with the paraxial description of the Airy-Gauss beam. For a Gaussian laser beam with an optical wavelength $\lambda$ and a half beam width (at $1 / \mathrm{e}^{2}$ intensity) $w_{0}$, on which a cubic phase $\exp \left[\mathrm{i} \beta^{3}\left(x^{3} / 3+x y^{2}\right) / \sqrt{2}\right]$ with a scale factor $\beta$ is added and which is then focused by an optical element with an effective focal length $f$, the propagation depends on the transverse length scale factor $x_{0}=\sqrt{2} f \beta / k$, the dimensionless longitudinal coordinate $\zeta=2 z /\left(k x_{0}^{2}\right)$, and the confinement factor $a=1 /\left(w_{0}^{2} \beta^{2}\right)$ [12]. Here, $k=2 \pi n / \lambda$, with $n$ being the refractive index of the medium. The resulting line focus given by the mainlobe of the complex electric field envelope [8],

$$
\begin{aligned}
E(\zeta, x, y)= & \operatorname{Ai}\left(\frac{x-y}{x_{0}}-\frac{\zeta^{2}}{4}+\mathrm{i} a \zeta\right) \operatorname{Ai}\left(\frac{x+y}{x_{0}}-\frac{\zeta^{2}}{4}+\mathrm{i} a \zeta\right) \\
& \times \exp \left[a\left(\frac{2 x}{x_{0}}-\xi^{2}\right)-\mathrm{i} \xi\left(\frac{\xi^{2}}{6}+a^{2}+\frac{x}{x_{0}}\right)\right],
\end{aligned}
$$

follows a parabolic trajectory in the $x z$ plane with a quadratic coefficient of $q=1 /\left(k^{2} x_{0}^{3}\right)$ [see Fig. 1(b)]. At the vertex of the parabola, the effective radius is $r=k^{2} x_{0}^{3} / 2$, and the angle $\alpha$ between the optical axis and the line focus varies along $z$ as $\alpha(z)=\arctan (2 q z)$. Over a length of $l \approx 2 w_{0} f^{2} \beta^{3} / k$ the intensity of the mainlobe exceeds $1 / \mathrm{e}^{2}$ of its maximum.

We used a $2 \mathrm{f}$ setup for creating the Airy-Gauss beam, as shown in Fig. 1(a) and similar to [4], imaging the cubic phase produced by a diffractive optical element (DOE: Airy Beam Generator, Holo/Or) with a microscope objective (MO). As the front focal plane of the MO lies within the housing of the MO, the DOE has been placed as close to the $\mathrm{MO}$ as the mechanical parts allow (effective distance $4 \mathrm{~mm}$ ). The effect of the resulting additional quadratic phase at the back focal plane (Fourier plane) is negligible, as we obtained the expected propagation behavior of an Airy-Gauss beam as shown in Figs. 2(a) and 2(b). In contrast, placing the DOE at the (accessible) front focal plane of aspheric lenses leads to a much more distorted beam propagation in experiments.

The Amphos 200XHE laser $\left(\lambda=1030 \mathrm{~nm}, M^{2}=1.1\right.$, $\omega_{0}=2.65 \mathrm{~nm}$ ) used for micromachining can emit bursts of one to four pulses with a delay of $25 \mathrm{~ns}$ and a pulse duration $t_{p}$

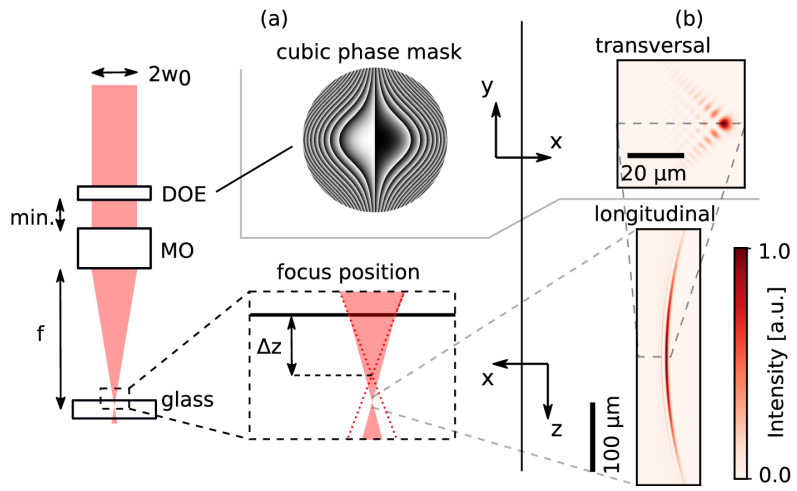

Fig. 1. Experimental 2f-setup (a) used for creating an Airy-Gauss beam (see text for details) and intensity profiles (b) according to Eq. (1) for $f=10 \mathrm{~mm}$. Throughout the paper, the focus position is given as the shift $\Delta z$ of the glass surface with respect to the focus position in air (red dotted line).

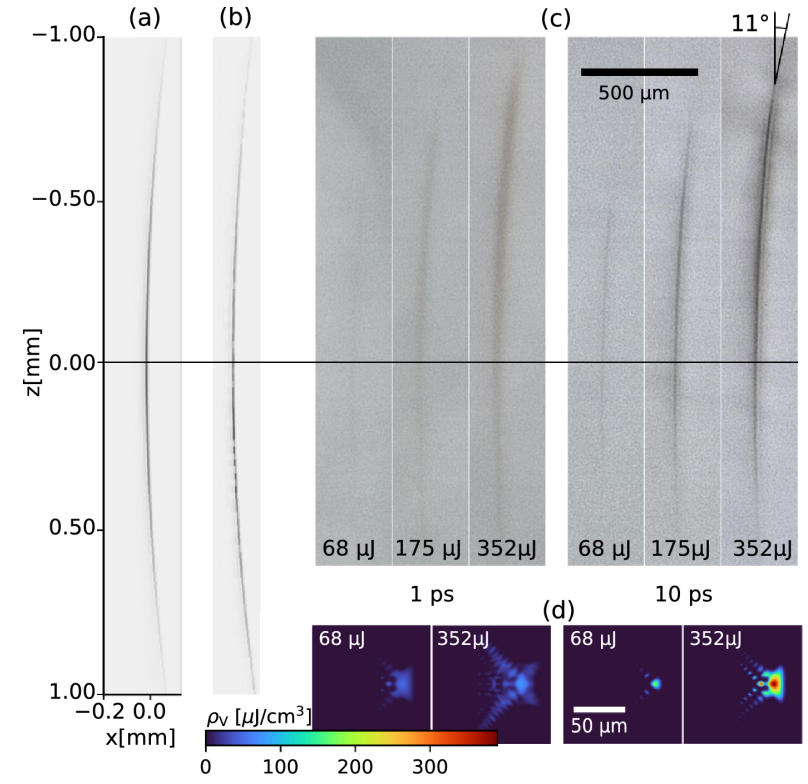

Fig. 2. Linear propagation of an Airy-Gauss beam with focal length $f=20 \mathrm{~mm}$ in air observed experimentally (b) agrees very well with the expected profile (a) given by Eq. (1). Light microscopy cross sections of permanent in-volume modifications in glass (c) also clearly follow the same trajectory. Corresponding deposited energy densities in the focal plane (d) obtained from numerical simulations confirm the higher confinement of deposited energy for long pulses. The $z$ scale of the profiles in air in (a), (b) is stretched by the refractive index of borosilicate glass ( $n=1.46)$ to mimic propagation in glass.

between 1 ps and 10 ps. The scale factor of the cubic phase $\beta$ was fixed at $3^{1 / 3} \mathrm{~mm}^{-1}$. Thus, with focal lengths of $10 \mathrm{~mm}$ and $20 \mathrm{~mm}$, an effective radius of curvature in air of $0.70 \mathrm{~mm}$ and $5.6 \mathrm{~mm}$ could be reached, respectively.

Sheets of borosilicate glass (SCHOTT Borofloat 33) of various thicknesses were placed horizontally on a motorized XY stage with the laser beam coming from the top. The orientation of the Airy beam with respect to the mechanical axes and the exact position of the focus, defined as the point of maximum intensity in linear propagation, was determined by examining the ablation pattern at the glass surface. The focus of the Airy beam was subsequently placed within the glass volume by decreasing the distance between the focusing optics and the glass sheet by a defined distance $\Delta z$ (see Fig. 1). The glass was then moved horizontally with respect to the laser beam while picking single laser bursts, resulting in discrete modifications, separated along the lateral translation direction by a constant distance, a so-called pitch, of 10,25 , or $50 \mu \mathrm{m}$. This translation direction was oriented perpendicularly to the acceleration direction of the beam, thus maximising the curvature of the cross section profile. Experiments were performed for various combinations of pulse duration, burst mode, pulse energy, focus position, and pitch.

To first examine the pure volume modification, we placed the focus deeply within a $6.5 \mathrm{~mm}$ thick glass sheet $(\Delta z=2 \mathrm{~mm})$, thus avoiding plasma ignition at the surface. We inspected the longitudinal profile of the volume modifications by cutting the glass perpendicularly to the abovementioned lateral translation direction, polishing it, and looking down this translation direction using reflected light microscopy (Keyence VHX 6000). For these profile measurements, we took special care to avoid double shots. We found that the volume modifications show the 
expected curvature for a wide range of experimental parameters (see Fig. 2). With increasing pulse energy, the trajectory remains unchanged, while the length and lateral extent of the modifications increase, for $f=20 \mathrm{~mm}$ reaching lengths of up to $2 \mathrm{~mm}$ and a maximum angle $a_{\max }=11^{\circ}$. For 1 ps pulses, the modifications remain broad and indistinct. More confined and stronger modifications are observed for longer pulse durations. The experimental profiles show a shift of the maximum intensity or damage with respect to the vertex of the trajectory, probably partly due to a slight misalignment of the laser beam [14]. In particular, however, we observe an increasingly asymmetric damage distribution along the parabola with increasing pulse energy, with the most extensive damage shifted towards the laser source and a long tail away from it.

To increase our understanding of the material damage, we simulated the energy deposition by using a unidirectional pulse propagation model accounting for all relevant nonlinear propagation effects, in particular, the laser-generated conduction electrons $[15,16]$. The preferential energy deposition in front of the linear focus observed in the experiments can also be seen in our simulation results (see Supplement 1). It can be explained by Kerr self-focusing and nonlinear absorption due to plasma generation, in particular, in the sidelobes of the Airy beam. While the total energy deposition was larger for short pulses with $t_{\mathrm{p}}=1 \mathrm{ps}$, the energy density was much higher for the longer pulses with $t_{\mathrm{p}}=10 \mathrm{ps}$ for all pulse energies considered [see Fig. 2(d)]. For short pulses, the laser affected zone is much broader due to intensity clamping $[17,18]$, resulting in high absorption, but a low energy confinement. This corroborates the experimental observation of more confined and stronger modifications for the 10 ps pulses in Fig. 2(c), and indicates that longer pulses are favorable for cutting applications. However, avalanche ionization is known to be dominant compared to multiphoton ionization for long pulses, leading to a less deterministic process [19], disadvantageous for the intended process.

So far, only bulk modifications were considered, and any surface modifications were excluded. The threshold for laser induced volume damage is supposed to be higher than that for the surface [20]. Indeed, we determined the surface damage threshold intensity for Borofloat 33 as $(1.07 \pm 0.12) \mathrm{TW} / \mathrm{cm}^{2}$ for $t_{\mathrm{p}}=10 \mathrm{ps}$, similar to previous values for borosilicate glass [21]. On the other hand, for a peak intensity of $2.4 \mathrm{TW} / \mathrm{cm}^{2}$ (theoretical, linear propagation), we hardly see any volume modification even for long pulses in Fig. 2(c), 10 ps, $68 \mu \mathrm{J}$. For the 2D Airy in our experiments, the ratio between main- and sidelobe intensity is only about two, compared to above five for a Bessel beam. In previous Airy beam micromachining studies, this was not an issue, because the sidelobes were propagating in air [4], but when focusing within the glass volume, the balance between surface and volume damage is of particular importance due to this low focal contrast.

To study the relationship between surface and volume modifications for the Airy beam in more detail, we chose a focus position closer to the surface of the sample $(\Delta z=200 \mu \mathrm{m})$ and observed the resulting modifications with both reflected and transmitted light microscopy (Zeiss Axio Imager). By changing the peak intensity while keeping the fluence constant, we monitored surface and bulk damage for various pulse configurations, shown in Fig. 3. For this we also considered a burst mode operation of the laser, as this can enhance the laser energy deposition

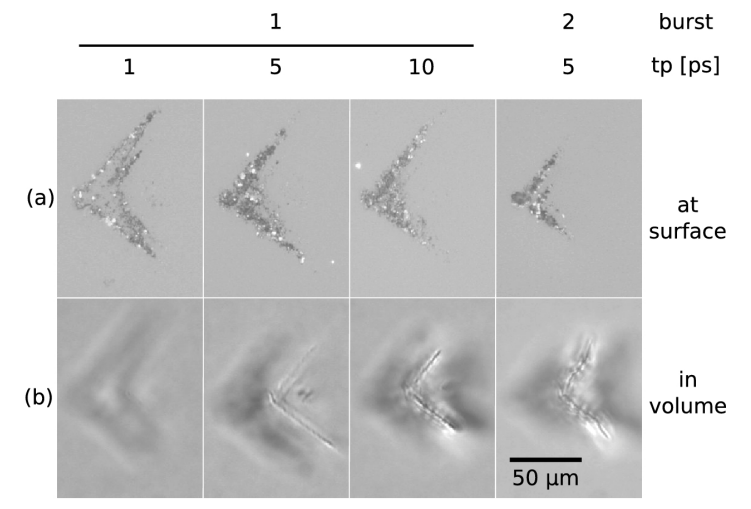

Fig. 3. Top views of permanent modifications (a) at the surface and (b) at a depth of $60 \mu \mathrm{m}$ within the volume of the glass with focal length $f=10 \mathrm{~mm}$, burst energy $E_{\text {burst }}=204 \mu \mathrm{J}$, and focus position $\Delta z=200 \mu \mathrm{m}$ for varying pulse durations $t_{p}$ and burst configurations. Both (a) reflected and (b) transmitted light microscopy were used for optimal contrast; still the blurred image of the surface modification can be seen in (b).

in the bulk [1]. We could obtain a strong mechanical damage within the volume, even close to the surface, for $t_{p}=5 \mathrm{ps}$ and a burst with two pulses. Longer pulses, on the other hand, show weaker volume modifications close to the surface, even though there is significant surface damage. In fact, we suspect that the plasma ignited at the surface may even lead to a shadowing effect, suppressing volume modifications close to the surface.

We note that our pulse durations lie in the intermediate range where the scaling of the surface damage threshold changes from a more intensity dominated regime for short pulse durations to a more fluence dominated regime for long pulse durations [22]. It may well be that a different scaling with intensity or fluence between surface and volume damage is the main reason for the observed optimum at intermediate pulse durations and burst.

For the separation experiments, we used glass of $525 \mu \mathrm{m}$ thickness, allowing perforation with $f=10 \mathrm{~mm}$. The glass pieces were subsequently separated by either mechanical breaking (cleaving) or etching with potassium hydroxide [23,24] (see Supplement 1). The resulting profile was recorded using confocal light microscopy in addition to the cross section analysis. Although it was possible to cleave the glass along a line perforated with the Airy beam, the new surface follows only partially the perforations created by the laser process: often the crack "cuts short" and runs through the convex side, building up as concoidally fractured bits within the concave side of the intended cut. The process then rather resembles laser scribing [25] rather than proper full volume cutting.

In contrast, Fig. 4 shows the fully expressed convex side obtained after separation by etching after laser processing with an Airy beam with $f=10 \mathrm{~mm}$, using a burst of two pulses with each a width of $t_{\mathrm{p}}=5 \mathrm{ps}$ and energy of $E_{\text {burst }}=228 \mu \mathrm{J}$, perforating with a pitch of $10 \mu \mathrm{m}$. The resulting overlap between the sidelobes of adjacent modifications increases the local energy deposition, but we do not expect a large impact on curvature of the convex edge, as the sidelobes are pointing to the concave side. The convex profile follows a parabola with an effective radius of curvature $r_{\text {eff }}=774 \mu \mathrm{m}$, which is smaller than the radius of the Airy mainlobe trajectory $(r=1.5 \mathrm{~mm})$. This stronger curvature and the large top angle $a_{\max }=18^{\circ}$ can be understood as an extra taper angle resulting from the etching 


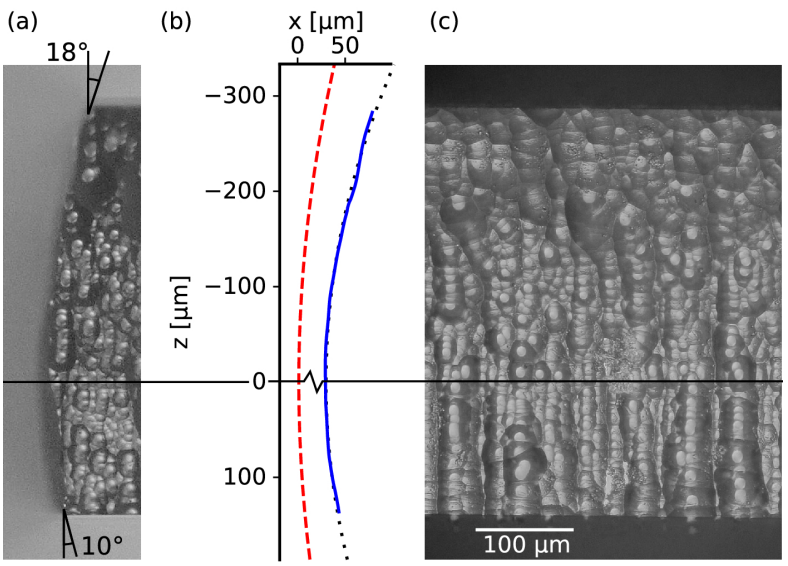

Fig. 4. Result of cutting a $525 \mu \mathrm{m}$ thick glass sheet by perforation with an Airy beam and subsequent etching. A cross section view (a) parallel to the line of laser modifications shows the curved profile of the cut. The average height profile (b) measured by confocal light microscopy (solid blue) and its parabolic fit (dotted black) exhibit a considerably stronger curvature than the Airy profile (dashed red), which can be attributed to the etching process. The side view onto the convex side (c) shows a coarser structure at the bottom, probably due to less extensive damage, leading to more localized etching.

process adding to the curved Airy profile. Due to the preferential absorption in the upper part of the glass sheet (as discussed above) we had to choose a focus position below the center of the glass sheet $(\Delta z \approx 220 \mu \mathrm{m})$ to be able to perforate the glass sheet completely. The side view in Fig. 4(c) corroborates more extensive damage in the upper part of the glass sheet and weaker damage in the lower part, as the edge surface below the focus shows a coarser structure. This coarser structure can be explained by a more localized etching process due to less extensive (and more variable) laser damage.

We note that the opposite side of the cut (not shown here) does not exhibit the expected concave profile after etching. Instead, more material is removed on the upper side of the glass sheet, thus resulting in a nearly planar, inclined edge. We attribute this finding to an increased removal rate during etching in the glass volume modified by interactions with the Airy sidelobes in addition to the previously mentioned taper angle.

In conclusion, we have demonstrated single pass perforation of glass sheets of $525 \mu \mathrm{m}$ thickness with an Airy-Gauss beam, yielding separation with a convex edge after etching. As for other cutting processes, the choice of laser parameters is crucial for the separability of the cut. However, the low focal contrast of the Airy beam renders the balance between surface and volume damage particularly important. By means of a combined experimental and numerical study, we identified suitable parameters for confined bulk energy deposition and moderate surface damage, in particular, with respect to the sidelobes of the Airy profile. The etching process necessary for separation further reduced the achievable radius of curvature by almost a factor of two, well beyond that of the Airy trajectory, which itself is limited by the trade-off between focus length and curvature. We expect that this first report of single pass curved glass cutting will trigger further research activities on micromachining with accelerating beams, aiming for thicker glass and more pronounced curvatures. Promising alternative candidates for producing curved modifications could be accelerating Bessel beams [26,27] or other accelerating beams with optimized intensity distribution [28].

Funding. GENCI (A0070506129, A0080507594); Qatar National Research Fund (NPRP 12S-0205-190047); SCHOTT AG.

Disclosures. DS: SCHOTT AG (E, P), JUT: SCHOTT AG (E, P), SS: SCHOTT AG (F).

Data Availability. Data underlying the results presented in this paper are not publicly available at this time but may be obtained from the authors upon reasonable request.

Supplemental document. See Supplement 1 for supporting content. REFERENCES

1. K. Mishchik, R. Beuton, O. D. Caulier, S. Skupin, B. Chimier, G. Duchateau, B. Chassagne, R. Kling, C. Hönninger, E. Mottay, and J. Lopez, Opt. Express 25, 33271 (2017).

2. K. Bergner, M. Müller, R. Klas, J. Limpert, S. Nolte, and A. Tünnerman, Appl. Opt. 57, 5941 (2018).

3. M. Jenne, D. Flamm, K. Chen, M. Schäfer, M. Kumkar, and S. Nolte, Opt. Express 28, 6552 (2020).

4. A. Mathis, F. Courvoisier, L. Froehly, L. Furfaro, M. Jacquot, P. A. Lacourt, and J. M. Dudley, Appl. Phys. Lett. 101, 071110 (2012).

5. A. Marcinkevičius, S. Juodkazis, S. Matsuo, V. Mizeikis, and $H$. Misawa, Jpn. J. Appl. Phys. 40, L1197 (2001).

6. M. K. Bhuyan, F. Courvoisier, P.-A. Lacourt, M. Jacquot, L. Furfaro, M. J. Withford, and J. M. Dudley, Opt. Express 18, 566 (2010).

7. M. V. Berry and N. L. Balazs, Am. J. Phys. 47, 264 (1979).

8. G. A. Siviloglou, J. Broky, A. Dogariu, and D. N. Christodoulides, Phys. Rev. Lett. 99, 213901 (2007).

9. Z. Yang, M. Prokopas, J. Nylk, C. Coll-Lladó, F. J. Gunn-Moore, D. E. K. Ferrier, T. Vettenburg, and K. Dholakia, Biomed. Opt. Express 5, 3434 (2014).

10. P. Panagiotopoulos, D. G. Papazoglou, A. Couairon, and S. Tzortzakis, Nat. Commun. 4, 2622 (2013).

11. L. Froehly, F. Courvoisier, A. Mathis, M. Jacquot, L. Furfaro, R. Giust, P. A. Lacourt, and J. M. Dudley, Opt. Express 19, 16455 (2011).

12. P. Polynkin, M. Kolesik, J. V. Moloney, G. A. Siviloglou, and D. N. Christodoulides, Science 324, 229 (2009).

13. N. K. Efremidis, Z. Chen, M. Segev, and D. N. Christodoulides, Optica 6, 686 (2019).

14. Y. Hu, G. A. Siviloglou, P. Zhang, N. K. Efremidis, D. N. Christodoulides, and Z. Chen, Nonlinear Photonics and Novel Optical Phenomena, Vol. 170 of Springer Series in Optical Sciences, (Springer, 2012), pp. 1-46.

15. L. Bergé, S. Skupin, R. Nuter, J. Kasparian, and J. P. Wolf, Rep. Prog. Phys. 70, 1633 (2007)

16. O. Dematteo Caulier, K. Mishchik, B. Chimier, S. Skupin, A. Bourgeade, C. Javaux Léger, R. Kling, C. Hönninger, J. Lopez, V. Tikhonchuk, and G. Duchateau, Appl. Phys. Lett. 107, 181110 (2015).

17. W. Liu, S. Petit, A. Becker, N. Aközbek, C. Bowden, and S. Chin, Opt. Commun. 202, 189 (2002)

18. A. Schmitt-Sody, H. G. Kurz, L. Bergé, S. Skupin, and P. Polynkin, New J. Phys. 18, 093005 (2016).

19. N. Sanner, O. Utéza, B. Chimier, M. Sentis, P. Lassonde, F. Légaré, and J. C. Kieffer, Appl. Phys. Lett. 96, 071111 (2010).

20. N. Bloembergen, Appl. Opt. 12, 661 (1973).

21. D. Nieto, J. Arines, G. M. O'Connor, and M. T. Flores-Arias, Appl. Opt. 54, 8596 (2015).

22. B. C. Stuart, M. D. Feit, S. Herman, A. M. Rubenchik, B. W. Shore, and M. D. Perry, Phys. Rev. B 53, 1749 (1996).

23. J. Gottmann, J. Laser Micro/Nanoeng. 8, 15 (2013).

24. D. Flamm, D. Grossmann, M. Kaiser, J. Kleiner, M. Kumkar, K. Bergner, and S. Nolte, in Proc. Lasers in Manufacturing Conference (2015), Vol. 253.

25. S. Nisar, L. Li, and M. A. Sheikh, J. Laser Appl. 25, 042010 (2013).

26. J. Zhao, P. Zhang, D. Deng, J. Liu, Y. Gao, I. D. Chremmos, N. K. Efremidis, D. N. Christodoulides, and Z. Chen, Opt. Lett. 38, 498 (2013).

27. I. D. Chremmos and N. K. Efremidis, Phys. Rev. A 88, 063816 (2013).

28. S. Barwick, Opt. Lett. 36, 2827 (2011). 